Service social

\title{
La garde partagée en famille recomposée : conditions facilitantes et conséquences possibles
}

\section{Claudette Guilmaine}

Volume 39, numéro 3, 1990

Familles recomposées après divorce

URI : https://id.erudit.org/iderudit/706501ar

DOI : https://doi.org/10.7202/706501ar

Aller au sommaire du numéro

\section{Éditeur(s)}

École de service social de l'Université Laval

ISSN

1708-1734 (numérique)

Découvrir la revue

Citer cet article

Guilmaine, C. (1990). La garde partagée en famille recomposée : conditions facilitantes et conséquences possibles. Service social, 39(3), 64-79.

https://doi.org/10.7202/706501ar

\section{Résumé de l'article}

Dans la réorganisation des relations parents-enfants après une séparation ou un divorce, plusieurs modalités de garde des enfants sont possibles. Certaines peuvent devenir plus difficiles après une recomposition familiale. L'article présente les résultats d'une recherche exploratoire auprès de parents et d'enfants utilisant la garde partagée ainsi que des conclusions d'une pratique au Service de médiation familiale en Cour supérieure. L'auteure propose huit conditions, chez les trois ou quatre adultes en cause, qui facilitent le succès de cette modalité de garde d'enfants. Elle ajoute cinq facteurs concrets habituellement associés à la réussite, et critique les énoncés de certains auteurs. Enfin, elle donne quelques conséquences possibles, en gains et en difficultés, pour les enfants et les parents. 


\section{La garde partagée en famille recomposée : conditions facilitantes et conséquences possibles}

Claudette Guilmaine

La garde partagée et la famille recomposée sont deux réalités de la société contemporaine. Bien que ces phénomènes relativement nouveaux soient de plus en plus fréquents ${ }^{1}$, nos connaissances sont limitées sur ces sujets. Et elles le sont encore plus lorsque les deux réalités sont vécues dans une même expérience familiale.

Le présent article est destiné à mettre en relief les conditions qui semblent favoriser une application harmonieuse de la garde partagée en famille recomposée. En plus de nuancer certains facteurs généralement associés au succès de la garde partagée, je tenterai d'éclairer les conséquences possibles de l'arrivée d'un nouveau conjoint pour le parent et l'enfant en garde partagée.

Une recherche exploratoire menée auprès de parents expérimentant cette modalité de garde m'a permis de systématiser et d'approfondir mes connaissances dans ce domaine ${ }^{2}$. De plus, ma pratique professionnelle au service d'expertise pour la Cour supérieure a enrichi ma réflexion d'une expérience concrète auprès de parents séparés ou en famille recomposée.

Le présent article constitue donc une exploration de cette double réalité de la garde partagée en famille recomposée dans une optique d'intervention adaptée.

\section{La réorganisation familiale}

Après une séparation ou un divorce, la famille est toujours vivante; elle prend toutefois des formes différentes, elle se réorganise autrement (Ahrons, 1987). 
La question de la garde des enfants est souvent cruciale au moment de la séparation. Quand les deux parents sont " adéquats » ( $c^{\prime}$ est-à-dire qu'ils ont des capacités parentales suffisantes pour assurer le bien-être de l'enfant) et qu'ils désirent tous les deux maintenir ou développer leur engagement auprès de l'enfant, la garde partagée devient une solution intéressante pour eux et pour ce dernier.

La garde partagée est une modalité de garde où l'enfant a deux domiciles principaux et habite en alternance avec son père et sa mère dans un partage de temps qui peut varier d'une proportion de 1/3-2/3 à 1/2-1/2 (Abarbanel, 1979). Ainsi, les deux parents de l'enfant peuvent jouer pleinement leur rôle auprès de lui et assumer l'ensemble des responsabilités parentales. Quant à l'enfant, il peut garder un lien affectif continu et ancré dans le quotidien avec chacun d'eux.

Une image (élitiste) des parents capables d'appliquer une garde partagée avec succès se dégage souvent des écrits touchant cette modalité de garde. L'analyse des propos de parents qui la vivent révèle, par ailleurs, que plusieurs maintiennent la garde partagée sans toutefois considérer leurs conditions d'application comme étant " idéales ". Il est d'ailleurs frappant que la garde unique à la mère a longtemps été ordonnée par les tribunaux sans qu'aucune condition y soit associée. Par contre, l'expérience a démontré que certaines attitudes maternelles, dont l'obstruction systématique à la relation père-enfant, ont eu des répercussions négatives déplorables pour chacun des membres de la famille.

Certains auteurs, dont Abarbanel (1979) et Luepnitz (1982), ont vu dans la recomposition familiale un risque pour la garde partagée. En effet, la relation du nouveau conjoint avec les enfants de l'autre n'est pas forcément aussi « idyllique " que la relation de couple.

Par contre, Irving (1984), dans sa recherche, constate que la recomposition familiale n'est pas nécessairement un obstacle à la garde partagée. Si le parent est très proche de ses enfants et désire poursuivre son engagement auprès d'eux, il est plus que probable que le choix du nouveau conjoint tienne compte de cette dimension.

\section{Conditions facilitantes}

Voyons maintenant quelles sont les conditions qui semblent faciliter un heureux " mariage » entre la garde partagée et la famille recomposée.

1. Que le nouveau conjoint soit favorable à la garde partagée.

2. Qu'il établisse une relation positive avec les enfants et ne fasse pas obstacle à la relation parents-enfant. 
3. Que l'ex-conjoint accepte "minimalement " la présence du nouveau conjoint auprès de ses enfants. Que l'enfant ait la permission affective de se lier à cette personne et à la famille de celle-ci.

4. Qu'une communication "fonctionnelle "s'établisse entre les deux noyaux familiaux sans utiliser les enfants comme messagers.

5. Que les adultes des deux systèmes se respectent et respectent la vie privée de chacun.

6. Que les adultes ne soient pas en conflit profond (sans nécessairement être amis).

7. Qu'ils fassent preuve de maturité et de souplesse.

\section{Être favorable à la garde partagée}

Tout comme pour les parents qui optent pour la garde partagée, la motivation du nouveau conjoint à la vivre est importante, car cette formule comporte ses exigences.

Un parent ou un nouveau conjoint qui ne voudrait pas assumer la présence des enfants la moitié du temps (ou presque) pourrait se sentir très frustré et en faire payer le prix aux enfants ou à l'autre parent. II est très facile de trouver des prétextes au conflit et aux revendications, que les enfants soient en garde unique ou en garde partagée.

Un intérêt sincère pour l'enfant et le désir de s'impliquer auprès de lui sont donc des éléments facilitateurs comme l'ont souligné plusieurs auteurs : Benedek et Benedek (1979), Elkin (1984), Steinman (1981), Volgy et Everett (1985). Des motivations de garde trop centrées sur les avantages financiers, sur un prestige social accru (pour les pères), un désir de vengeance face à l'autre parent, une lutte de pouvoir, un essai d'effacer le passé ou d'annuler l'autre parent... seraient à dénoncer.

En garde partagée, il arrive qu'un parent soit moins motivé que l'autre, qu'il soit inquiet ou résiste. Ilfeld et al. (1982), de même que Volgy et Everett (1985), ont constaté qu'après une période d'expérimentation de la formule des parents peuvent se montrer très satisfaits même s'ils ne désiraient pas la garde partagée au départ. Un essai peut aider des pères à faire la preuve de leurs capacités parentales et des mères à déléguer une partie des responsabilités et des tâches qu'elles se réservaient durant la vie commune. Ces " gardes partagées à l'essai » peuvent sécuriser les parents. Ainsi en est-il dans certaines situations de recomposition familiale où la garde partagée a été acceptée, mais non choisie par le nouveau conjoint. 


\section{Établir une relation positive avec les enfants sans faire obstruc- tion à la relation parents-enfant}

Les liens créés par la garde partagée peuvent devenir très significatifs mais le contraire existe aussi, c'est-à-dire des conjoints peu motivés à assumer une garde partagée des enfants de l'autre et qui " subissent " la situation sans vraiment y trouver d'avantages et sans que leur relation avec les enfants se développe positivement. Ce contexte d'application se révèle pénible pour tous. Ceux qui s'en accommodent sont probablement ceux qui limitent leurs attentes à une " cohabitation pacifique".

II semble que la motivation du nouveau conjoint soit également tributaire de la sympathie et de l'attirance ressenties envers les enfants. Certains nouveaux conjoints se sentent forcés d'aimer les enfants de l'autre ou se font un devoir d'éveiller l'amour de ces enfants pour eux. D'autres croient que l'amour des enfants de l'autre naîtra instantanément. Ce mythe a été mentionné par Visher et Visher (1978), Capaldi et McRae (1979) et Nelson et Nelson (1982). Des attentes plus limitées aident à dédramatiser les réactions d'opposition et d'adaptation du début où chacun tente de définir sa nouvelle place et de conserver ses acquis sur le plan affectif.

Il est inévitable et " normal " que la relation de chaque conjoint avec ses enfants biologiques soit différente de la relation qu'il a avec les enfants de l'autre conjoint, quoique l'amour identique pour les enfants constitue une autre illusion à laquelle des parents s'accrochent. Comme le souligne Marie-Christine Saint-Jacques (1991), ces mythes sont nuisibles au fonctionnement des familles, quel que soit le modèle de garde ${ }^{3}$. Même si les " comparaisons ", les incompréhensions et les discussions semblent inscrites dans le quotidien de plusieurs parents, Duberman (1973) évalue comme positives les relations entre les beaux-parents et les enfants de son étude.

Lorsque les enfants du nouveau conjoint n'ont pas le même modèle de garde que les enfants de l'autre parent, d'autres ajustements s'imposent pour établir des règles de fonctionnement claires et équitables. Les conjoints doivent aussi composer avec la dimension affective de cette présence ou de cette absence de leurs enfants à certains moments donnés.

II arrive que le nouveau conjoint souhaite réparer en quelque sorte ce qu'il peut avoir vécu comme un échec ou une désillusion dans la famille précédente. La tentation le guette alors de prendre la place du " parent absent " auprès des enfants de son partenaire pour recommencer en oubliant le passé. Cette attitude peut faire violence en niant l'héritage de chacun. 
La discipline s'avère particulièrement difficile à exercer quand le beau-père s'accapare cette fonction avant d'avoir établi avec l'enfant une relation affective satisfaisante. Selon Hetherington (1987), le risque que l'enfant rejette le beau-parent est présent dans ces cas de " sur-engagement autoritaire " comme dans les situations de désengagement du beau-parent.

Un autre écueil surgit lorsque le nouveau conjoint est jaloux du passé et de l'intimité que le parent et ses enfants souhaitent parfois retrouver. La complicité qui existait dans la cellule monoparentale peut, par contre, être préservée par des activités ou du temps privilégié parent-enfant si le nouveau conjoint est capable de composer avec leur besoin légitime de se retrouver entre eux sans sa présence.

\section{Accepter l'autre et donner à l'enfant la permission de l'aimer}

Les parents qui appliquent la garde partagée se disent d'abord motivés par le mieux-être des enfants. Selon Greif (1979), cette préoccupation du mieux-être des enfants est suffisante, si elle est bien réelle, pour assurer le succès de la garde partagée. Les parents veulent que les enfants aient accès à leurs deux parents et qu'ils profitent de l'apport de chacun sans avoir à choisir. L'enfant reçoit alors le message implicite ou explicite qu'il a le droit d'aimer ses deux parents même si ceux-ci ne s'aiment pas assez pour rester ensemble.

L'arrivée de nouveaux conjoints vient parfois menacer cet équilibre affectif. Un parent peut, surtout s'il est encore sans conjoint, vivre difficilement le fait que son ex-conjoint en famille recomposée lui apparaisse bénéficier de tout ce dont il est privé pour sa part. L'idéalisation aidant, l'autre peut être perçu comme heureux, sans problème et profitant en plus d'une famille biparentale et d'une relation conjugale.

Il est important que l'enfant « sente » qu'il ne trahit pas un parent $s^{\prime}$ il éprouve de l'affection pour le conjoint de son autre parent. Le " conflit de loyauté » est particulièrement cruel pour un enfant qui ne veut déplaire ni à l'un ni à l'autre de ses parents et qui reçoit des messages négatifs les concernant ou concernant leurs conjoints. II est également lourd pour l'enfant de se sentir responsable du bonheur du parent perçu comme victime.

Pour que l'enfant dispose de ce "visa " affectif, ses parents devront se situer eux-mêmes en "pays libre ". Sans cette paix intérieure, le parent ne pourra donner la permission affective à son enfant de se relier à l'autre. Cela est plus facile quand le deuil émotionnel est réalisé ou en voie de l'être. Selon Capaldi et McRae (1979), le fait 
que le deuil de la famille précédente soit résorbé facilite grandement l'adaptation de chacun. II est, par contre, plus sain et aidant pour l'enfant d'entendre un message authentique où le parent avoue ses limites quant à l'acceptation du nouveau conjoint plutôt que de percevoir une contradiction entre le "dit " et le "non-dit", car on ne peut nier que l'acceptation de l'autre se révèle parfois très exigeante pour le parent sur le plan affectif.

\section{Être capable de communiquer de façon "fonctionnelle " sans utiliser les enfants}

Des auteurs comme Rothberg (1983) et Ricci (1980) soulignent I'importance d'une communication directe. Eder (1978) et Elkin (1984) précisent que le dialogue doit être ouvert au moins en ce qui concerne les enfants. II faut toutefois noter que des parents ont vu leur communication s'améliorer par l'application de la garde partagée. Derdeyn et Scott (1984) mettent en relief que la collaboration peut être suscitée par la garde partagée.

Les exigences de communication reliées à la garde partagée sont toutefois moindres qu'on le croit parfois. Les conversations régulières, les discussions au restaurant, les visites aux domiciles, les téléphones quotidiens sont loin d'être la norme. II ressort des propos de plusieurs parents qu'il peut être suffisant et même plus confortable émotivement de restreindre les communications à la sphère parentale en évitant les sujets conflictuels. Certains utilisent un cahier d'information qui circule avec les enfants d'un domicile à l'autre. $\mathrm{D}^{\prime}$ autres se téléphonent sur leurs lieux de travail lorsqu'une décision importante s'impose pour les enfants. Le domaine financier demeure à risque : un partage équitable des frais relatifs aux enfants peut diminuer les discussions et les conflits.

À cet égard, l'arrivée de nouveaux conjoints peut relancer des débats et modifier des règles préalablement établies entre les parents. L'exercice de clarification des besoins et des attentes gagne à être fait ou refait.

Finalement, il est un principe de communication à sauvegarder : ne pas utiliser les enfants comme messagers entre les adultes. Même s'il est exigeant émotivement d'exprimer clairement à l'autre parent les attentes et les demandes concernant l'enfant, cette attitude de maturité libère l'enfant $d$ 'une lourde responsabilité et de dilemmes au cœur desquels il serait piégé. Sans parfois le réaliser, un parent peut demander à l'enfant de faire preuve de plus de courage et d'affirmation qu'il en a lui-même à l'égard de l'autre parent, qu'il s'agisse de réclamer un changement $d^{\prime}$ horaire ou un remboursement de frais... 


\section{Se respecter, se faire confiance, ne pas s'ingérer}

Les deux systèmes parentaux gagnent à établir des frontières claires. Le nouveau couple a besoin d'un domaine privé et cette intimité se doit d'être respectée tant par les enfants que par l'autre parent.

La garde partagée peut ébranler ces frontières, qu'il s'agisse de visites impromptues des enfants pour récupérer un vêtement ou un jouet, ou encore d'appels téléphoniques ou d'intrusions fréquentes de l'autre parent.

Plus les adultes se feront confiance dans leurs capacités parentales et se respecteront comme personnes ou comme membres de "nouveaux couples", plus la vie sera harmonieuse. L'importance de cette confiance et de cette reconnaissance des capacités de l'autre parent est mise en évidence par Volgy et Everett (1985), Durst et al. (1985), de même que par Benedek et Benedek (1979).

Des règles peuvent être établies pour respecter le caractère privé de chacune des familles. À titre d'exemple, il peut être aidant de préciser l'usage d'une clé du domicile de l'autre ou les heures d'appel, etc. Mais par-delà les règles, l'attitude de respect et l'image transmise aux enfants ont une grande influence.

Cela ne signifie pas par ailleurs que les deux milieux de vie de l'enfant doivent observer des règles de fonctionnement identiques. Les parents parlent de "consensus sur les valeurs de base ", mais de différences dans l'application quotidienne. Abarbanel (1979) et Volgy et Everett (1985) mentionnent comme condition de succès la « capacité de tolérer les différences». Ahrons (1987) utilise l'expression " agree to disagree ». Ernst et Altis (1981) soulignent, quant à eux, la possibilité d'accord sur des règles parentales même s'il existe des désaccords par ailleurs.

\section{Ne pas nourrir de conflits profonds... sans nécessairement être amis}

C'est Steinman (1981) qui indique l'absence de conflit profond comme condition à la garde partagée. D'autres auteurs ou chercheurs précisent qu'il est possible de séparer l'hostilité conjugale des obligations parentales (Roman et Haddad, 1978; Ahrons, 1987). Des témoignages de parents nous incitent à croire qu'une certaine collaboration est possible même s'il existe des tensions et des conflits. Monsieur le juge Mayrand (1988) parle de "rééquilibrer l'autorité parentale". C'est d'ailleurs dans ce sens que, comme experts à la Cour supérieure, nous en venons parfois à recommander ce que j'appellerais des " gardes partagées stratégiques ". II s'agit alors d'encourager la fin d'une lutte de pouvoir et un engagement équivalent des deux parents en les invitant à se centrer sur l'intérêt de l'enfant. 
De tels aménagements sont fragiles, mais gagnent parfois à être tentés. L'état actuel de la recherche ne nous permet toutefois pas d'évaluer si le conflit a plus d'effet sur les enfants en garde partagée qu'en garde unique (Joan Kelly, 1987). Il est évident que plus l'enfant est mêlé au conflit, plus il en subit les répercussions. Nous savons, par contre, que la qualité de la relation entre les parents peut être améliorée grâce à la garde partagée (Morgenbesser et Nehls, 1981). II est intéressant de constater que cette modalité de garde peut diminuer le conflit, selon Grote et Weinstein (1977) et selon Fortin (1985).

L'attitude d'un nouveau conjoint sera donc déterminante dans les cas litigieux. Si celui-ci fait alliance avec le parent pour alimenter le conflit avec l'ex-conjoint en utilisant les enfants, le risque d'échec est élevé. Certains parents, même s'ils sont tous les deux en familles recomposées, continuent parfois de s'espionner, de compétitionner ou de se harceler, ce qui constitue une façon de demeurer en lien. Si, par contre, le nouveau conjoint laisse les parents négocier tout ce qui concerne les enfants sans s'interposer et $s^{\prime}$ il favorise une approche consensuelle, son attitude peut contribuer à une détente du climat qui favorise même la garde partagée.

Irving (1984), dans sa recherche à grand échantillon, fait toutefois ressortir qu'il n'est pas nécessaire que les parents soient "amis " pour réussir la garde partagée. Il faut que les parents puissent maintenir un certain contact (Rothberg, 1983), mais plus importante encore semble cette capacité de séparer les zones " conjugales » et " parentales" (Durst et al., 1985; Elkin, 1987). À ce sujet, mon étude exploratoire illustre trois types de relation postrupture : une relation parentale sans amitié mais sans ingérence de l'ex-domaine conjugal, une relation parentale sans amitié mais avec ingérence et une relation parentale avec amitié et sans ingérence. Le premier type de relation est présenté comme très satisfaisant pour les parents.

\section{Faire preuve de maturité, être responsable et souple...}

Les écrits portant sur les conditions associées à la garde partagée mettent l'accent sur certaines qualités. La flexibilité est mentionnée par Abarbanel (1979), Benedek et Benedek (1979) et Ernst et Altis (1981), la capacité de faire des compromis par Steinman (1981), l'équilibre personnel par Elkin (1987), la stabilité personnelle et émotionnelle de chaque parent et le sens des responsabilités par Volgy et Everett (1985).

Il faut toutefois éviter d'idéaliser les parents capables d'appliquer une garde partagée. La recomposition familiale, en intégrant de nouveaux membres, complexifie nécessairement les relations et l'organisation concrète de la garde partagée (ex. : les horaires, les vacances, 
les activités parascolaires, les visites dans les familles élargies, les Fêtes, etc.). Les exigences en termes de "compromis" et de "souplesse "sont accrues. Les besoins à satisfaire sont plus nombreux, mais on peut toutefois compter sur les ressources et les forces nouvelles nées de cette restructuration. De plus, les enfants ont une grande capacité d'adaptation et peuvent retirer des gains de leur double appartenance. S'ils sont soutenus en ce sens, ils bénéficieront des richesses de chacun des milieux et de ces nouveaux modèles d'identification ou de relation.

\section{Précisions complémentaires}

Afin de parfaire ce balisage, j'apporterai à l'aide de la littérature et de ma pratique quelques nuances concernant des conditions habituellement associées à la garde partagée :

- la proximité des domiciles;

- I'aisance financière;

- la flexibilité d'horaire de travail;

- la coparentalité prérupture;

- et l'âge scolaire des enfants.

Bien que la proximité des domiciles facilite les déplacements et le maintien du réseau social des enfants, et qu'il serait impossible de considérer cette modalité si les parents habitaient à une trop grande distance géographique (Ernst et Altis, 1981), certains parents s'accommodent des transports. Ils s'assurent ainsi de ce que J. Thomas (1985) appelle la "distance idéale ", celle où ils n'ont pas à se voir sans l'avoir planifié. Ce confort émotionnel peut également orienter le choix du lieu de résidence après la recomposition familiale. Comme le précisent Folberg et Graham (1979), Irving (1984) et Elkin (1987), la proximité physique est facilitante mais non indispensable.

Une certaine sécurité financière est également aidante selon Benedek et Benedek (1979) et Hagen (1987), mais Irving (1984) affirme que cette formule n'est pas accessible uniquement aux riches. Parmi les parents que j'ai rencontrés, plusieurs optent pour transporter les effets des enfants plutôt que de faire les achats en double.

Pour ce qui est de la flexibilité d'horaire de travail soulignée comme condition par Volgy et Everett (1985), elle facilite sans contredit la présence des parents auprès des enfants pour les rendezvous scolaires, médicaux ou autres, mais il ressort des propos de plusieurs usagers que l'attitude de disponibilité est plus importante que l'horaire de travail comme tel. 
Si certains auteurs et chercheurs considèrent comme conditions à la garde partagée la forte implication du père avant la rupture (Steinman, 1981) et le lien significatif parent-enfant avant la rupture (Volgy et Everett, 1985), d'autres croient à la possibilité de développer ce lien après la rupture (Roman et Haddad, 1978; Wallerstein et Kelly, 1980). Selon Greif (1979) et Irving (1984) un apprentissage de rôle est possible.

Parmi les parents que j'ai rencontrés, certains voyaient dans la garde partagée l'occasion pour les pères d'investir davantage dans le temps consacré à leur enfant et de raffermir les liens. Les mères se disaient prêtes à partager les responsabilités et à céder une part du contrôle, tandis que les pères désiraient s'impliquer davantage.

Il est toutefois indéniable que si la coparentalité a existé durant la vie commune en matière de partage des responsabilités et des tâches parentales, la transition vers la garde partagée en est facilitée et le niveau de satisfaction des parents est habituellement plus élevé (Goldsmith, 1980).

Dans certains milieux plus traditionnels, des pères sont encouragés à déléguer leurs responsabilités parentales à leur nouvelle conjointe. On voit, par ailleurs, de plus en plus de pères assumant la garde partagée sans conjointe ou conservant après la réorganisation familiale leur domaine de responsabilités initiales. Cela secoue nos conceptions sexistes et illustre un changement social manifeste.

La dernière condition est encore très controversée. Certains estiment que les enfants doivent être au moins d'âge scolaire pour pouvoir composer adéquatement avec deux milieux de vie (Eder, 1979). Irving (1984) a lui aussi mis en doute la pertinence de la garde partagée pour de très jeunes enfants.

Des parents ont appliqué la garde partagée avec des enfants de 0 à 2 ans en privilégiant la formule $\mathrm{du}$ " nid ", où ce sont les parents qui changent de domicile alors que le bébé conserve le même milieu physique. D'autres ont adopté des rythmes plus courts, par exemple aux deux jours, pour tenir compte de la façon dont les jeunes enfants expérimentent le «temps».

Certains résultats d'études laissent penser que, contrairement à ce que l'on peut craindre, les enfants en garde partagée ne présentent pas de confusion quant à leur mode de vie dans la mesure où l'horaire offre une certaine régularité (Steinman, 1981). Nous avons toutefois peu de données quant aux conséquences de l'alternance de résidence sur les très jeunes enfants.

II m'apparaît, par ailleurs, que la notion de " stabilité " gagnerait à être revue dans le contexte social actuel. En effet, l'enfant dont les parents sont sur le marché du travail a souvent à s'adapter à plusieurs milieux et à plusieurs figures de référence. Qu'il s'agisse de familles 
«biparentales » ou «monoparentales », l'enfant est confié pour certaines périodes de temps à plus $\mathrm{d}^{\prime} \mathrm{un}$ autre adulte. On parle alors de la "stabilité affective», de la " permanence des figures sécurisantes». Peut-être faut-il poser le même regard lorsqu'il s'agit de la garde partagée!

II semble donc que le défi de la garde partagée pour de jeunes enfants consiste à leur assurer une forme de stabilité sur les plans psychologique et affectif par la présence régulière des parents et des autres personnes significatives, de même que des points de repère fixes dans les lieux, dans l'horaire de vie et grâce à certains objets chéris par l'enfant.

\section{Conséquences possibles}

Je résumerai maintenant quelques conséquences de cette réorganisation familiale que constitue la famille recomposée, mentionnées comme possibles par des parents en garde partagée (voir tableau en annexe).

Les gains qu'ils perçoivent pour les enfants sont les suivants : l'apport d'une figure féminine auprès du père, la relation positive dont l'enfant peut ainsi profiter avec un autre adulte, une modification positive de la dynamique fraternelle (diminuant, par exemple, la rivalité dans la fratrie) et le fait que la famille recomposée soit vue comme répondant davantage à la "normalité " sociale.

Les gains que les parents considèrent pour eux-mêmes sont le fait que, pour certains pères, ce soit la solution à l'incapacité de fonctionner seul, la hausse du niveau de vie, de même que le soutien et la réponse aux besoins affectifs de l'adulte. Le parent qui profite de la présence d'un autre adulte sent parfois, par ricochet, ses attentes diminuer en ce qui a trait à l'apport affectif de ses enfants.

Les difficultés que peut entraîner pour l'enfant l'arrivée d'un nouveau conjoint sont, $d$ 'après les parents, la peur de perdre sa place et la réaction de possessivité qui s'ensuit parfois. De plus, dans les situations conflictuelles de recomposition familiale, des parents mentionnent la peur de l'enfant de vivre une deuxième rupture et son espoir ravivé que les parents se réconcilient. D'autres parents relèvent également des difficultés relationnelles entre l'enfant et le nouveau conjoint et les tensions que cela suscite. Le parent témoin de ces conflits vit souvent un déchirement dans sa double loyauté à ses enfants et au nouveau conjoint.

En ce qui concerne les difficultés vécues par les parents, ceux-ci constatent que la recomposition familiale peut faire obstacle à la communication entre les parents appliquant la garde partagée, plus par- 
ticulièrement lorsque le nouveau conjoint est possessif ou se sent menacé par la relation entre les ex-conjoints. Établir une distinction entre l'ex-domaine conjugal et le domaine parental actuel s'avère très important pour mieux délimiter les frontières. Parfois, la recomposition familiale peut réduire la disponibilité personnelle à offrir aux enfants, du moins au début. De plus, l'arrivée d'un nouveau conjoint oblige à des contacts entre celui-ci et l'autre parent, ce qui peut être très exigeant sur le plan émotif. Le parent vivant seul doit, par surcroît, composer avec le fait que son enfant passe la moitié de son temps avec le nouveau conjoint de l'autre, ce qui peut accroître son sentiment de perte, de solitude ou d'envie. En dernier lieu, la recomposition familiale nécessite parfois un déménagement avec toutes les conséquences que cela entraîne quant au choix du quartier, de l'école, etc.

En résumé, les parents interrogés mettent en relief que la recomposition familiale exige de multiples adaptations pour chacun. Ces parents n'ont toutefois jamais remis en question leur choix de garde partagée à cause de nouveaux conjoints vivant avec eux ou avec leur ex-conjoint.

\section{Conclusion}

La garde partagée et la famille recomposée sont de nouvelles réalités sociales mal balisées où chacun est l'artisan de son modèle et où une intervention professionnelle judicieuse peut mobiliser positivement les énergies et faciliter l'ajustement de chacun.

L'adaptation des adultes et des enfants après la séparation ou le divorce des parents est un processus long et complexe (Wallerstein et Kelly, 1980). Hess et Camara (1979) ont mis en relief I'importance de la qualité de la relation des parents sur l'ajustement postdivorce des enfants et, plus encore, l'importance de la qualité de la relation de l'enfant avec chacun de ses parents. Ces balises peuvent guider les décisions et les attitudes parentales et encourager la coparentalité postrupture.

La recomposition familiale constitue, elle aussi, un changement " stressant " qui nécessite des adaptations multiples pour chacun. Cet événement et l'anxiété qu'il fait naître peuvent réveiller des conflits non résolus et rouvrir des blessures mal cicatrisées. II s'agit, par ailleurs, d'une autre occasion de " dédramatiser » certaines difficultés passagères inhérentes à la réorganisation physique matérielle et affective. Le fait de mieux cerner les gains et les difficultés possibles rattachés à l'arrivée d'un nouveau conjoint peut parfois faciliter l'adaptation. 
Diane Germain (1986) rappelle aux parents d'avoir des attentes réalistes et de faire le «deuil de l'idéal " et de la famille intacte. Je crois que c'est dans cette mesure que la famille recomposée pourra intégrer la garde partagée sans trop de heurts.

La recherche, l'intervention de groupe auprès de cette clientèle, l'approche de type "médiation », de même que le partage des expertises professionnelles, m'apparaissent comme autant de moyens pour parfaire et enrichir notre travail professionnel face à ces changements sociaux.

\section{ANNEXE*}

Conséquences possibles, pour la garde partagée, de la présence d'un nouveau conjoint

\begin{tabular}{|c|c|}
\hline Gains pour I'enfant & Gains pour le parent \\
\hline $\begin{array}{l}\text { Figure féminine auprès du père } \\
\text { Relation positive avec un autre } \\
\text { adulte } \\
\text { Modification positive de la dy- } \\
\text { namique fraternelle } \\
\text { "Normalité » du modèle }\end{array}$ & $\begin{array}{l}\text { Solution à l'incapacité de fonc- } \\
\text { tionner seul } \\
\text { Hausse du niveau de vie } \\
\text { Soutien et réponse aux besoins } \\
\text { affectifs }\end{array}$ \\
\hline Difficultés pour l'enfant & Difficultés pour le parent \\
\hline $\begin{array}{l}\text { Possessivité et peur de perdre } \\
\text { sa place } \\
\text { Si conflit, } \\
\text { Peur de vivre une deuxième } \\
\text { rupture } \\
\quad \text { ou } \\
\text { Espoir que les parents se récon- } \\
\text { cilient } \\
\text { Difficultés relationnelles } \\
\text { nouveau conjoint-enfant }\end{array}$ & $\begin{array}{l}\text { Obstacle à la communication } \\
\text { entre les parents } \\
\text { Disponibilité réduite } \\
\text { Exigence émotive des contacts } \\
\text { entre nouveau conjoint et autre } \\
\text { parent } \\
\text { Nécessité d'un déménagement }\end{array}$ \\
\hline
\end{tabular}

* Tirée du mémoire de l'auteure. 


\section{Notes}

${ }^{1}$ Richard Cloutier, Louise Careau et Jacques Drolet (1987) évaluent que $25 \%$ des parents optent pour une garde partagée après leur séparation.

${ }^{2}$ L'auteure a récemment publié un livre à l'intention des parents et des intervenants sous le titre de La garde partagée, un heureux compromis.

${ }^{3}$ À ce sujet, voir son article présenté dans ce même numéro de la revue Service social.

\section{Références bibliographiques}

Abarbanel, Alice (1979). "Shared Parenting after Separation and Divorce : A Study of Joint Custody ", American Journal of Orthopsychiatry. vol. 49, $\mathrm{n}^{\circ} 2$.

AHrons, Constance R. et Roy H. RodGers (1987). "Strengthening Binuclear Families » : 201-220, dans Divorced Families. New York, London : Ed. Nortons Company.

BenedeK, E. et R. BenedeK (1979). " Joint Custody : Solution or Illusion ? ", American Journal of Psychiatry, $136: 1540-1544$.

CAPALDI, Frederick et Barbara MCRAE (1979). Step Families : A Cooperative Responsability. New York : New Viewpoints/Vision Books.

Cloutier, Richard, Louise Careav et Jacques Drolet (1988). Communication présentée dans le cadre du congrès 1989 de la Corporation des psychologues du Québec. École de psychologie, Université Laval.

DerdeYN, André P., et Elizabeth ScotT (1984). " Joint Custody : A Critical Analysis and Appraisal ", American Journal of Psychiatry, vol. 54, n 2 : 199-207.

DUBERMAN, Lucile (1973). "Step-kin relationship ", Journal of Marriage and the Family, vol. 35, $\mathrm{n}^{\circ} 2$ : 287-292.

Durst, P.L., N.V. WedemeYer et L.A. ZurCher (1985). "Parenting Partnership after Divorce : Implications for Practice", Social Work, vol. 30, n 5 : 423-428.

EDER, Vicki (1978). "Shared Custody : An Idea Whose Time Has Come », Conciliation Courts Review, vol. 16, $\mathrm{n}^{\circ} 1$ (reproduit dans Joint Custody, a Handbook for Judges, Lawyers and Counselors - The Association Family Conciliation Courts, Florida, 1979).

ElKIN, Meyer (1987). " Joint Custody : Affirming that Parents and Families are Forever ", National Association of Social Workers. Janvier-février : 18-23.

ELKIN, Meyer (1984). "Joint Custody : A Self-Determined Structure for Shared Parenting ", Counciliation Courts Review, vol. 22, $\mathrm{n}^{\circ} 2$.

ERNST, Theodore et Ruth ALTIS (1981). "Joint Custody and Co-parenting : Not by Law but by Love ", Child Welfare, vol. LX, nº $10: 669$.

FolberG, Jay H. et Marva Graham (1979). "Joint Custody - Myth and Reality, Presumption Superiority of Sole Custody », University of California, Law Review, $\mathrm{n}^{\circ} 12$.

FORTIN, Denise (1985). L'entente de garde conjointe suite aux interventions du service de médiation à la famille de Montréal. Université de Montréal, août. 
Germain, Diane (1986). "Famille reconstituée : le deuil de l'idéal », dans P. Gauthier, Les nouvelles familles. Montréal : Éditions Saint-Martin.

GoldSMITH, Jean (1980). "Relationship between Former Spouses Descriptive Findings ", Journal of Divorce, vol. 42, $\mathrm{n}^{\circ} 2$.

Greif-Brown, Judith (1979). "Fathers, Children and Joint Custody ", American Journal of Orthopsychiatry, vol. 49, $\mathrm{n}^{\circ} 2$ (avril).

Grote, D. et J. Weinstein (1977). "Joint Custody: A Viable and Ideal Alternative ", Journal of Divorce, vol. $1, \mathrm{n}^{\circ} 1$.

Guilmaine, Claudette (1989). L'expérience de la garde partagée : étude exploratoire auprès de parents, sous la direction de Marie Simard, École de service social, Université Laval.

Guilmaine, Claudette (1990). "La garde partagée : recherche exploratoire ", Intervention, $\mathrm{n}^{\circ}$ 85, mars : 84-91.

GuILMAINE, Claudette (1991). La garde partagée : un heureux compromis. Montréal : Éditions Stanké, Coll. Parcours.

HaGeN, Jan L. (1987). "Proceed with Caution : Advocating Joint Custody ». National Association of Social Workers. Janvier-février : 26-30.

Hess, R.D. et K.A. Camara (1979). "Post-divorce Family Relationship as Mediating Factors in the Consequences of Divorce for Children ". Journal of Social Issues, vol. 35, $\mathrm{n}^{\circ} 4: 79-96$.

HetherinCtON, Mavis E. (1987). «Family Relations Six Years after Divorce » dans P. Kay et I. Tallman (éd.), Remarriage and Stepparenting. Current Research and Theory. New York : Guilford Press.

Ilfeld, F.N., H.Z. Ilfeld et T.R. AleXander (1982). "Does Joint Custody Work ? A First Look at Outcome Data of Relitigation ", American Journal of Psychiatry, $139: 62-66$.

Irving, H.H., M. Benjamin et N. Trocme (1984). "Shared Parenting : an Empirical Analysis Utilizing a Large Data Base », Family Process, vol. 23, n 4 : 561-569.

Kelly, Joan B. (1987). Long Term Adjustment in Children of Divorce : Converging Findings and Implications for Practice. Revised version of an invited address presented at the Annual Meeting of the American Psychological Association, New York.

LUEPNITZ, Deborah Anna (1982). Child Custody: A Study of Families after Divorce. Toronto : Lexington Books.

MAYRAND, Juge A. (1988). "La garde conjointe, rééquilibrage de l'autorité parentale ", Revue du Barreau canadien, 67 : 193-228.

Morgenbesser, Mel. et Nadine Nehts (1981). Joint Custody: An Alternative for Divorcing Families. Chicago : Nelson Hall, p. 166.

Nelson, Margaret et Gordon K. Nelson (1982). "Problems of equity in the reconstituted family: a social exchange analysis", Family Relations, vol. $31: 223-231$.

RıcCl, Isolina (1980). Mom's House, Dad's House: Making Shared Custody Work. New York : MacMillan.

RocKLIN, Joanne (1984). Joint Custody and Sole-Custody Mothers : The Effect of the Coparental Relationship and Sex-Role Attitudes on Satisfaction with Change in Maternal Influence. California School of Professional Psychology. Los Angeles, P.H.D., DAI V45, 12 section B, P3959.

Roman, Melvin et William Haddad (1978). The Disposable Parent : The Case for Joint Custody. New York : Holt, Rinehart and Winston. 
RothBeRG, B. (1983). "Joint Custody : Parental Problems and Satisfactions", Family Process, vol. $22: 43-52$.

SAINT-JACQUES, Marie-Christine (1990). Familles recomposées : qu'avons-nous appris au fil des ans? Service social, vol. 39, $\mathrm{n}^{\circ} 3$.

SteinMAN, Suzan (1981). "The Experience of Children in a Joint Custody Arrangement : A Report of Study", American Journal of Orthopsychiatry, vol. 51 : 403-414.

THOMAS, Judith Wayne (1985). Co-Parenting after Divorce : Issues and Opportunities in a New Status, University Microfilm International, Columbia University, P.H.D. DAI V46, 3 section A, P808.

VISHER, Emily B. et John S. VISHER (1978). "Common Problems of Stepparents and Their Spouses", American Journal of Orthopsychiatry, vol. 48 : 252-262.

VolgY, Sandra S. et Craig A. EVERETT (1985). " Joint Custody Reconsidered : Systemic Criteria for Mediation », Journal of Divorce, 3-4 : 131-150.

WALLERSTEIN, Judith et Joan Kelly (1980). Surviving the Breakup : How Children and Parents Cope with Divorce. New York : Basic Books. 